\title{
Hepatite B e o status de soroconversão de auxiliares em saúde bucal na Atenção Primária - Uma associação do perfil imunológico e fatores influentes
}

\author{
Hepatitis B and the seroconversion status of oral health assistants in Primary Care - An association \\ of immunological profile and influencing factors
}

Hepatitis B y el estado de seroconversión de los auxiliares de salud bucal en Atención Primaria Una asociación de perfil inmunológico y factores influyentes

\section{Resumo}

Julia Arruda Batista

ORCID: https://orcid.org/ 0000-0001-9621-7201 Universidade Estadual Paulista "Júlio de Mesquita Filho", Brasil

E-mail: jarrudabaptista@gmail.com

Clea Adas Saliba Garbin

ORCID: https://orcid.org/0000-0001-5069-8812 Universidade Estadual Paulista "Júlio de Mesquita Filho", Brasil E-mail: clea.saliba-garbin@unesp.br

Bruno Wakayama

ORCID: https://orcid.org/0000-0002-5152-3683 Universidade Estadual Paulista "Júlio de Mesquita Filho", Brasil E-mail: brunowakayama@gmail.com

Tânia Adas Saliba

ORCID: https://orcid.org/0000-0003-1327-2913 Universidade Estadual Paulista "Júlio de Mesquita Filho", Brasil E-mail: tania.saliba@unesp.br Artênio José Saliba Garbin

ORCID: https://orcid.org/0000-0003-1566-681X Centro Universitário Católico Salesiano Auxilium, Brasil E-mail: tesjg@hotmail.com

Artênio José Isper Garbin

ORCID: https://orcid.org/0000-0002-7017-8942 Universidade Estadual Paulista "Júlio de Mesquita Filho", Brasil E-mail: artenio.garbin@unesp.br

Dimensionar as características sociodemográficas, o conhecimento sobre a Hepatite B, as práticas de autocuidado em saúde, bem como, a associação destas variáveis com o perfil imunológico de auxiliares em saúde bucal por meio de ensaios enzimáticos anti HBsAg e imunocromatografia. Trata-se de um estudo epidemiológico transversal, realizado na Atenção Primária em 40 municípios da região noroeste do Estado de São Paulo, atendidos pela DRS II. A coleta dos dados foi estruturada em etapas, sendo que na primeira foi empregado questionários semiestruturados autoaplicáveis, dimensionado em domínios demográficos, conhecimento e práticas de autocuidado em saúde. A segunda consistiu na análise da ficha de imunização dos participantes, seguido da verificação do status de imunização dos ASBs por meio de um ensaio imunocromatográfico rápido anti-HBsAG. A análise dos dados foi realizada no software Statistical Package for the Social Sciences, utilizando os testes qui-quadrado de Pearson, teste exato de Fisher, teste da razão da máxima verossimilhança e o teste de normalidade Kolmogorov-Smirnov ( $\mathrm{p}<0,05)$. Participaram do estudo 158 ASBs, sendo $94,9 \%$ do sexo feminino, cor da pele branca $(83,5 \%)$, casada $(65,8 \%)$ e ensino médio completo (45,6\%). Quanto ao conhecimento percebeu-se que grande parcela desconhecia sobre o VHB e que apesar de adotarem os protocolos de biossegurança, 58,2\% já se acidentaram com percutâneos. Além disto, $45,6 \%$ dos auxiliares não estavam imunizados. Conclui-se que uma expressiva parcela dos ASBs não estava imune à contaminação pela hepatite $\mathrm{B}$, sendo que as lacunas no conhecimento, comportamentos e condutas destes profissionais apresentaram influência sobre o status de imunização.

Palavras-chave: Hepatite B; Pessoal de saúde; Vacinas; Contenção de riscos biológicos.

\begin{abstract}
Scale sociodemographic characteristics, knowledge about Hepatitis B, self-care practices in health, as well as the association of these variables with the immunological profile of oral health assistants through anti-HBsAg enzymatic assays and immunochromatography. This is a cross-sectional epidemiological study carried out in Primary Care in 40 municipalities in the northwest region of the State of São Paulo, served by the DRS II. Data collection was structured
\end{abstract}


in stages, in the first stage, self-administered semi-structured questionnaires were used, dimensioned in demographic domains, knowledge and self-care practices in health. The second consisted of analyzing the participants' immunization records, followed by checking the immunization status of the ASBs using a rapid anti-HBsAG immunochromatographic assay. Data analysis was performed using the Statistical Package for the Social Sciences software, using Pearson's chi-square test, Fisher's exact test, maximum likelihood ratio test and the KolmogorovSmirnov normality test $(\mathrm{p}<0.05)$. A total of 158 ASBs participated in the study, $94.9 \%$ female, white $(83.5 \%)$, married $(65.8 \%)$ and having completed high school (45.6\%). As for knowledge, it was noticed that a large portion did not know about HBV and that despite adopting biosafety protocols, 58.2\% had already had accidents with percutaneous products. In addition, $45.6 \%$ of assistants were not immunized. It is concluded that a significant portion of ASBs was not immune to hepatitis B contamination, and the gaps in knowledge, behavior and behavior of these professionals influenced the immunization status.

Keywords: Hepatitis B; Health personnel; Vaccines; Containment of biohazards.

\section{Resumen}

Escalar características sociodemográficas, conocimientos sobre Hepatitis B, prácticas de autocuidado en salud, así como la asociación de estas variables con el perfil inmunológico de los auxiliares de salud bucal mediante ensayos enzimáticos anti-HBsAg e inmunocromatografía. Se trata de un estudio epidemiológico transversal, realizado en Atención Primaria en 40 municipios de la región noroeste del Estado de São Paulo, atendidos por el DRS II. La recolección de datos se estructuró en etapas, en la primera etapa se utilizaron cuestionarios semiestructurados autoadministrados, dimensionados en dominios demográficos, conocimientos y prácticas de autocuidado en salud. El segundo consistió en analizar los registros de inmunización de los participantes, seguido de verificar el estado de inmunización de los ASB mediante un ensayo inmunocromatográfico rápido anti-HBsAG. El análisis de los datos se realizó mediante el software Statistical Package for Social Sciences, utilizando la prueba de chi-cuadrado de Pearson, la prueba exacta de Fisher, la prueba de razón de máxima verosimilitud y la prueba de normalidad de KolmogorovSmirnov ( $\mathrm{p}<0,05)$. Un total de 158 ASB participaron en el estudio, 94,9\% mujeres, blancas $(83,5 \%)$, casadas $(65,8 \%)$ y con estudios secundarios completos $(45,6 \%)$. En cuanto al conocimiento, se notó que una gran parte desconocía sobre el VHB y que a pesar de adoptar protocolos de bioseguridad, el 58,2\% ya había tenido accidentes con productos percutáneos. Además, el 45,6\% de los asistentes no estaban vacunados. Se concluye que una parte importante de los ASB no fue inmune a la infección por hepatitis B, y las brechas en el conocimiento, comportamiento y comportamiento de estos profesionales influyeron en su estado de inmunización.

Palabras clave: Hepatitis B; Personal de salud; Vacunas; Contención de riesgos biológicos.

\section{Introdução}

A hepatite B é uma infecção causada pelo vírus VHB, que pode desencadear desde uma inflamação no tecido hepático até quadros mais severos de necrose hepatocelular (Garbin et al., 2020)

De acordo com a Organização Mundial de Saúde (OMS), o vírus da hepatite B é uma das principais causas de mortalidade e morbidade à nível mundial (Gomes et al.,2020). Estima-se que 2 bilhões de indivíduos estão infectados pela hepatite B, e que cerca de 325 milhões convivem com a doença em sua forma crônica, sem diagnóstico e acesso aos tratamentos (Garbin et al., 2020). Diante disto, a hepatite B é considerada uma doença pandêmica, que apresenta índices epidemiológicos significativos, caracterizando-se como um grave problema de saúde pública (Garbin et al.,2018).

Dada a dimensão epidemiológica da hepatite B no cenário mundial, as entidades de saúde dispõem aos serviços e profissionais da área, diretrizes e recomendações a fim de alertar sobre os riscos inerentes da contaminação pelo vírus VHB, bem como, oportunizar a implementação de políticas públicas relacionadas à doença (World Health Organization,2015). No ano de 2017, um manual referente aos exames para diagnóstico da hepatite B foi publicado com o propósito de fortalecer as ações coordenadas em saúde, disponibilizando assim, o acesso aos testes laboratoriais para o grupo dos vulneráveis (World Health Organization, 2017).

Inseridos neste contexto, os profissionais da saúde estão mais susceptíveis à contaminação pelo vírus da hepatite B, em particular os cirurgiões-dentistas e auxiliares em saúde bucal, visto a proximidade com fluidos corporais, sangue e exposições percutâneas (Alavian et al., 2011). Embora inúmeros agentes patogênicos possam ser transmitidos pós-exposição acidental com instrumentais perfurocortantes, o VHB representa grande parcela das infecções decorrentes do contato com material biológico (Aldakhil et al., 2019). Dessa forma, algumas estratégias são empregadas a fim de prevenir e/ou conter a 
contaminação pelo VHB como uso de equipamentos de proteção individual (EPI) e a imunização ativa. Esta última é considerada um meio de controle e prevenção simples, seguro, eficaz e de maior impacto para a saúde individual e coletiva (Teixeira et al., 2020).

No Brasil, a vacina contra o VHB foi desenvolvida na década de 1970, e sua ampla distribuição na rede pública do país foi a partir de 1986, não apenas como uma forma de autocuidado na prática profissional, mas também uma ferramenta de proteção à integridade do paciente, contribuindo para redução da incidência da infecção (Garbin et al.,2020). Sendo assim, em 2017 a OMS propôs na Cúpula Mundial da Hepatite, a Declaração de São Paulo, na qual o país reafirma seu compromisso em adotar uma abordagem multidimensional para apoiar e implementar as principais intervenções definidas pela Estratégia Global acerca das Hepatites Virais, tendo como premissa a redução de novas infecções e a mortalidade em decorrência da hepatite B até o ano de 2030. (Yuan et al.,2019).

Apesar da vacina ter uma alta imunogenicidade e ser uma das formas de proteção mais eficaz contra o vírus da hepatite B, cerca de 10\% dos indivíduos não desenvolvem a imunidade protetora (Garbin et al., 2020; Resende et al., 2010). Outrossim, faz-se necessário pelos profissionais da saúde, o monitoramento do status da imunização mediante a observância dos níveis de anticorpos por meio do teste anti-HBs, sendo essa uma informação essencial para avaliar a necessidade de doses de reforço (Wakayama et al., 2019; Garbin et al., 2020; Souza et al., 2015; Jouneghani et al., 2017).

Assim, tendo em vista a expressiva concentração da força de trabalho dos auxiliares em saúde bucal na Atenção Primária em Saúde (APS) e da escassez de pesquisas que mapeiam o status de proteção vacinal e a prevalência da hepatite B neste grupo vulnerável, este estudo teve como objetivo dimensionar as características sociodemográficas, o conhecimento sobre questões relacionadas a Hepatite $\mathrm{B}$, as práticas de autocuidado em saúde, bem como, a associação destas variáveis com o perfil imunológico de auxiliares em saúde bucal por meio de ensaios enzimáticos anti HBsAg e imunocromatografia.

\section{Metodologia}

\section{Design do Estudo}

Trata-se de um estudo epidemiológico transversal, quantitativo (Marconi \& Lakatos, 2003) conduzido com Auxiliares em Saúde Bucal atuantes na Atenção Primária em Saúde (APS) em 40 municípios (774.441 habitantes) da região Noroeste do Estado de São Paulo, atendidos pelo Departamento Regional de Saúde II (DRS-II).

O Estado de São Paulo localiza-se na região Sudeste do Brasil e compreende o maior número de profissionais da área da saúde do país. Administrativamente, o estado é dividido em 17 Secretarias Regionais de Saúde (São Paulo, 2006) a fim de atender às diretrizes do Sistema Único de Saúde (SUS) e as reformas pactuadas pelos entes federados (Pacto pela Saúde) (Portaria nº 3999/2006) (Brasil,2006).

De acordo com os dados fornecidos pela DRS-II, no período da coleta, haviam 179 Auxiliares em Saúde Bucal ativos no serviço público. Todos os profissionais que aceitaram participar da pesquisa assinaram o Termo de Consentimento Livre e Esclarecido (TCLE). Foram excluídos os municípios nos quais os gestores não autorizaram a condução do estudo e os Auxiliares em Saúde Bucal que estavam afastados de suas atividades e/ou licença maternidade, aposentados, e aqueles que não estavam presentes após duas visitas no período da pesquisa.

\section{Coleta dos Dados}

A coleta dos dados foi dimensionada em duas etapas. Na primeira etapa, foi empregado questionários semiestruturados autoaplicáveis, elaborado para o estudo após levantamento bibliográfico, para inclusão das variáveis válidas, sensíveis e inferenciais. O instrumento foi dividido em domínios: características sociodemográficas; representatividade do 
conhecimento profissional sobre a hepatite B; e práticas de autocuidado em saúde dos participantes.

Previamente foi realizado um estudo piloto a fim de verificar a aplicabilidade e operabilidade do instrumento para atender ao objetivo da pesquisa. A aplicação do instrumento foi feita no local de trabalho dos participantes, sem atrapalhar e/ou interferir na organização e agendamentos de consultas.

$\mathrm{Na}$ segunda etapa, os Auxiliares em Saúde Bucal, de forma individual, foram encaminhados para uma área privativa disponibilizada nas unidades de saúde. Neste momento, foi solicitada a ficha de imunização dos participantes para verificação da completude do esquema vacinal (três doses) e foi inquerido aos profissionais sobre o teste de soroconversão anti-HBs anterior e seu resultado. Além disso, nesta fase do estudo, foi analisado o status de imunização dos ASBs por meio de um ensaio imunocromatográfico rápido anti- HBsAg ( ${ }^{\circledR}$ (Wama Diagnostica, São Carlos, SP, Brasil). Todas as normas de biossegurança preconizadas foram seguidas, garantindo a integridade física dos participantes e a sensibilidade e confiabilidade dos testes.

O dispositivo empregado para a verificação do status da imunização fundamenta-se na tecnologia imunocromatográfica e utiliza o princípio sanduíche para que haja a detecção de anticorpos anti-HBsAg no plasma, sangue total e/ou soro humano. No primeiro momento, o sangue foi coletado por meio da punção digital com auxílio de uma lanceta descartável e estéril. Em seguida, $100 \mu \mathrm{L}$ de sangue foi depositado no poço da amostra do dispositivo e lido 15-20 minutos após o início do ensaio. A leitura do resultado positivo, indicando que o indivíduo esta imunizado contra o VHB, ocorre quando anticorpos de superfície (anti-HBs) presente na amostra agrega-se ao conjugado HBsAg-ouro coloidal, dando origem ao complexo antígeno-anticorpo. Este complexo se desloca para área de teste (T), onde se liga ao antígeno HBsAg evidenciando uma faixa rosa avermelhada. O restante do complexo flui para a área controle (C) onde se liga aos anticorpos anti-HBsAg, aparecendo assim uma segunda faixa rosa avermelhada na região, determinando o status de imunização. O resultado negativo indica a não imunização do indivíduo ou níveis insuficientes de anticorpos $(<10 \mathrm{mIU} / \mathrm{mL})$. Essa condição se confirma quando apenas uma faixa rosa avermelhada é exibida na área de controle (C), enquanto que na área teste $(\mathrm{T})$ nenhuma reação é desencadeada (faixa rosa).

\section{Análise dos Dados}

Para a tabulação e análise dos dados foi utilizado o pacote estatístico Statistical Package for the Social Sciences (SPSS for Windows, versão 22.0, IBM, Armonk, New York, USA), e realizada análise bivariada por meio do teste qui-quadrado de Pearson, teste exato de Fisher e teste da razão da máxima verossimilhança, considerando valor de $\mathrm{p}<0,05$ para verificar associações entre as variáveis dependentes e independentes.

As variáveis quantitativas (idade, tempo de atuação como auxiliar em saúde bucal, anos no serviço público e número de erros), foi aplicado o teste de normalidade de Kolmogorov-Smirnov ( $\mathrm{p} \leq 0,05)$. Contudo, como o teste não atendeu ao objetivo proposto, utilizou-se o teste não paramétrico de Mann-Whitney, considerando o nível de significância de 5\% ( $\mathrm{p} \leq 0,05)$.

\section{Aspectos éticos e legais}

O presente estudo foi aprovado pelo Comitê de Ética e Pesquisa (CAAE: 54227416.0.0000.5420) e cumpriu todos os princípios éticos para a pesquisa com seres humanos exigidos na resolução 466/2012 do Conselho Nacional de Saúde do Brasil.

\section{Resultados}

Fizeram parte do universo amostral 158 Auxiliares em Saúde Bucal. Foi verificado que 54,4\% dos ASBs receberam pelo menos três doses da vacina e relataram que estavam imunizados contra o vírus da Hepatite B. 
Em relação ao perfil sociodemográfico a maioria dos participantes era do sexo feminino (94,9\%), cor da pele branca $(83,5 \%)$, casada $(65,8 \%)$ e completaram o ensino médio $(45,6 \%)$ (Tabela 1$)$.

Tabela 1. Distribuição das frequências e análise bivariada entre os fatores sociodemográficos e variável dependente.

\begin{tabular}{|c|c|c|c|c|c|c|c|}
\hline \multirow{3}{*}{ Variáveis } & \multicolumn{4}{|c|}{ Imunização } & \multirow{2}{*}{\multicolumn{2}{|c|}{ Total }} & \multirow{3}{*}{ p-valor } \\
\hline & \multicolumn{2}{|c|}{ Sim } & \multicolumn{2}{|c|}{ Não } & & & \\
\hline & $\mathbf{n}$ & $\%$ & $\mathbf{n}$ & $\%$ & $\mathbf{n}$ & $\%$ & \\
\hline \multicolumn{8}{|l|}{ Sexo } \\
\hline Masculino & 5 & 6,3 & 3 & 3,8 & 8 & 5,1 & $0,720 * *$ \\
\hline Feminino & 75 & 93,8 & 75 & 96,2 & 150 & 94,9 & \\
\hline \multicolumn{8}{|l|}{ Etnia } \\
\hline Branco & 62 & 77,5 & 70 & 89,7 & 132 & 83,5 & \\
\hline Negro & 2 & 2,5 & 3 & 3,8 & 5 & 3,2 & $0,038^{*}$ \\
\hline Pardo & 16 & 20,0 & 5 & 6,4 & 21 & 13,3 & \\
\hline \multicolumn{8}{|l|}{ Estado civil } \\
\hline Não casado & 18 & 22,5 & 36 & 46,2 & 54 & 34,2 & \\
\hline Casado / União estável & 62 & 77,5 & 42 & 53,8 & 104 & 65,8 & $0,002 *$ \\
\hline \multicolumn{8}{|l|}{ Escolaridade } \\
\hline Superior & 27 & 33,8 & 8 & 10,3 & 35 & 22,2 & \\
\hline Técnico Enfermagem & 22 & 27,5 & 9 & 11,5 & 31 & 19,6 & $0,000 *$ \\
\hline Técnico Saúde Bucal & 14 & 17,5 & 6 & 7,7 & 20 & 12,7 & \\
\hline Ensino Médio & 17 & 21,3 & 55 & 70,5 & 72 & 45,6 & \\
\hline
\end{tabular}

* Teste do Qui-quadrado

** Teste exato de Fisher

Fonte: Autores.

Na Tabela 2 foi possível constatar que a média de idade dos participantes do estudo que não estavam imunizados contra o VHB e o tempo de trabalho como ASBs foi respectivamente, 43,60 e 13,39 anos. Outro dado relevante, pelo teste de MannWhitney (Tabela 3), foram encontradas associações entre a variável dependente (imunização) e o número de erros dos profissionais analisados em relação à dimensão "conhecimento", ratificando que os auxiliares em saúde bucal que não estavam imunizados apresentavam maiores lacunas sobre questões elementares da doença e riscos à que estão expostos em sua rotina clínica quando comparados com os profissionais que estavam imunes ao vírus VHB.

Tabela 2. Distribuição de frequências descritivas e teste de comparação.

\begin{tabular}{|c|c|c|c|c|c|c|}
\hline Variáveis & Imunização & $\mathbf{n}$ & Mediana & Média & $\begin{array}{l}\text { Desvio- } \\
\text { padrão }\end{array}$ & p-valor \\
\hline \multirow{2}{*}{ Idade } & Sim & 80 & 39,00 & 39,91 & 10,24 & \multirow{2}{*}{0,015} \\
\hline & Não & 78 & 45,00 & 43,60 & 10,42 & \\
\hline \multirow{2}{*}{$\begin{array}{l}\text { Tempo de trabalho como } \\
\text { ASB }\end{array}$} & Sim & 80 & 10,00 & 11,97 & 8,14 & \multirow{2}{*}{0,350} \\
\hline & Não & 77 & 11,00 & 13,39 & 9,03 & \\
\hline \multirow{2}{*}{ Tempo de serviço público } & Sim & 80 & 9,00 & 11,75 & 9,16 & \multirow{2}{*}{0,147} \\
\hline & Não & 77 & 11,00 & 13,43 & 8,49 & \\
\hline \multirow{2}{*}{ Número de erros } & Sim & 80 & 2,00 & 1,92 & 1,24 & \multirow{2}{*}{0,000} \\
\hline & Não & 78 & 5,00 & 5,13 & 1,43 & \\
\hline
\end{tabular}

Fonte: Autores

A Tabela 3 demonstra algumas lacunas no que tange ao conhecimento dos profissionais da saúde acerca de questões elementares sobre a Hepatite B e imunoprevenção. Foi observado que uma parcela considerável dos auxiliares em saúde bucal não obteve nenhuma orientação sobre a doença (32,1\%), sendo percebida pela associação significativamente estatística entre esta variável e a imunização dos indivíduos $(\mathrm{p}=0,000)$. Outra associação percebida foi que os profissionais que não estavam 
imunizados desconheciam sobre o agente etiológico da Hepatite $B(p=0,000)$ e não apresentavam uma percepção adequada dos potenciais riscos à que estão expostos no consultório $(\mathrm{p}=0,037)$.

No que se refere ao conhecimento dos profissionais sobre imunoprevenção, verificou-se que cerca $41,1 \%$ dos ASBs responderam de forma incorreta sobre as vias de prevenção para a Hepatite B. Também foram encontradas por meio da análise bivariada, associações estatisticamente significantes entre a variável dependente e as vias de transmissão e prevenção da doença $(\mathrm{p}=0,000)$. Ainda nesta dimensão, os profissionais responderam de forma incorreta sobre o tempo de duração da imunização pela vacina $(57,7 \%)$, número de doses para obtenção de um esquema vacinal completo $(69,2 \%)$ e 45,6\% afirmaram ter tomado número de doses inferior e/ou nem sequer lembravam de quantas doses eram necessárias para a obtenção do esquema vacinal completo. Além disto, 96,2\% desconheciam a necessidade de realização do teste anti-HBs para a certificação do status de imunização.

Tabela 3. Distribuição das frequências e análise bivariada entre os fatores relacionados ao conhecimento e variável dependente.

\begin{tabular}{|c|c|c|c|c|c|c|c|}
\hline \multirow{3}{*}{ Variáveis } & \multicolumn{4}{|c|}{ Imunização } & \multirow{2}{*}{\multicolumn{2}{|c|}{ Total }} & \multirow[t]{3}{*}{ p-valor } \\
\hline & \multicolumn{2}{|c|}{ Sim } & \multicolumn{2}{|c|}{ Não } & & & \\
\hline & $\mathbf{n}$ & $\%$ & $n$ & $\%$ & n & $\%$ & \\
\hline \multicolumn{8}{|l|}{ Recebeu orientação sobre hepatite } \\
\hline \multicolumn{8}{|l|}{ B? } \\
\hline Não & 2 & 2,5 & 25 & 32,1 & 27 & 17,1 & $0,000 *$ \\
\hline Sim & 78 & 97,5 & 53 & 67,9 & 131 & 82,9 & \\
\hline \multicolumn{8}{|l|}{$\begin{array}{l}\text { Participou de algum curso sobre o } \\
\text { tema, nos últimos dois anos? }\end{array}$} \\
\hline Não & 70 & 87,5 & 76 & 97,4 & 146 & 92,4 & $0,018^{*}$ \\
\hline Sim & 10 & 12,5 & 2 & 2,6 & 12 & 7,6 & \\
\hline \multicolumn{8}{|l|}{ Você conhece o agente etiológico } \\
\hline \multicolumn{8}{|l|}{ da Hepatite B? } \\
\hline Errou & 26 & 32,5 & 68 & 87,2 & 94 & 59,5 & $0,000 *$ \\
\hline Acertou & 54 & 67,5 & 10 & 12,8 & 64 & 40,5 & \\
\hline \multicolumn{8}{|l|}{ Sabe qual o risco da ASB contrair } \\
\hline \multicolumn{8}{|l|}{ Hepatite B na odontologia? } \\
\hline Não sabe & 12 & 15,0 & 47 & 60,3 & 59 & 37,3 & $0,000 *$ \\
\hline Sabe & 68 & 85,0 & 31 & 39,7 & 99 & 62,7 & \\
\hline \multicolumn{8}{|l|}{ Conhece as vias de transmissão? } \\
\hline Errou & 11 & 13,8 & 56 & 71,8 & 67 & 42,4 & $0,000 *$ \\
\hline Acertou & 69 & 86,3 & 22 & 28,2 & 91 & 57,6 & \\
\hline \multicolumn{8}{|l|}{ Conhece as vias de prevenção? } \\
\hline Errou & 10 & 12,5 & 55 & 70,5 & 65 & 41,1 & $0,000 *$ \\
\hline Acertou & 70 & 87,5 & 23 & 29,5 & 93 & 58,9 & \\
\hline \multicolumn{8}{|l|}{ A vacina é para sempre? } \\
\hline Errou & 18 & 22,5 & 45 & 57,7 & 63 & 39,9 & $0,000 *$ \\
\hline Acertou & 62 & 77,5 & 33 & 42,3 & 95 & 60,1 & \\
\hline \multicolumn{8}{|l|}{ Quantas doses têm a vacina? } \\
\hline Errou & 6 & 7,5 & 54 & 69,2 & 60 & 38,0 & $0,000 *$ \\
\hline Acertou & 74 & 92,5 & 24 & 30,8 & 98 & 62,0 & \\
\hline \multicolumn{8}{|l|}{ Quantas doses da vacina contra a } \\
\hline \multicolumn{8}{|l|}{ Hepatite B você tomou? } \\
\hline Três ou mais doses & 79 & 98,8 & 7 & 9,0 & 86 & 54,4 & $0,000 *$ \\
\hline Menos que 3 doses / Não sabe & 1 & 1,3 & 71 & 91,0 & 72 & 45,6 & \\
\hline \multicolumn{8}{|l|}{ O que significa o teste antihbs? } \\
\hline Errou & 71 & 88,8 & 75 & 96,2 & 146 & 92,4 & $0,079 *$ \\
\hline Acertou & 9 & 11,3 & 3 & 3,8 & 12 & 7,6 & \\
\hline \multicolumn{8}{|l|}{ Conhece o protocolo de acidente? } \\
\hline Não & 29 & 36,3 & 36 & 46,2 & 65 & 41,1 & $0,206^{*}$ \\
\hline Sim & 51 & 63,8 & 42 & 53,8 & 93 & 58,9 & \\
\hline
\end{tabular}

* Teste do Qui-quadrado.

Fonte: Autores. 
A Tabela 4 refere-se à alguns domínios relacionados aos comportamentos dos profissionais que podem ser fatores considerados intervenientes na produção de anticorpos. Diante disto, foram encontradas associações estatisticamente significantes entre o consumo de bebidas alcoólicas, ausência de prática de atividades físicas e o status da imunoprevenção $(\mathrm{p}<0,005)$.

Tabela 4. Distribuição das frequências e análise bivariada entre os comportamentos dos profissionais e variável dependente.

\begin{tabular}{|c|c|c|c|c|c|c|c|}
\hline \multirow{3}{*}{ Variáveis } & \multicolumn{4}{|c|}{ Imunização } & \multirow{2}{*}{\multicolumn{2}{|c|}{ Total }} & \multirow{3}{*}{ p-valor } \\
\hline & \multicolumn{2}{|c|}{ Sim } & \multicolumn{2}{|c|}{ Não } & & & \\
\hline & $\mathrm{n}$ & $\%$ & $\mathrm{n}$ & $\%$ & $\mathrm{n}$ & $\%$ & \\
\hline \multicolumn{8}{|l|}{ Você fuma? } \\
\hline Não & 76 & 95,0 & 69 & 88,5 & 145 & 91,8 & $0.135^{*}$ \\
\hline Sim & 4 & 5,0 & 9 & 11,5 & 13 & 8,2 & \\
\hline \multicolumn{8}{|c|}{ Você ingere bebida alcoólica? } \\
\hline Não & 63 & 78,8 & 41 & 52,6 & 104 & 65,8 & $0,001^{*}$ \\
\hline $\operatorname{sim}$ & 17 & 21,3 & 37 & 47,4 & 54 & 34,2 & \\
\hline \multicolumn{8}{|c|}{ Você pratica atividade física? } \\
\hline Não & 10 & 12,5 & 53 & 67,9 & 63 & 39,9 & $0,000^{*}$ \\
\hline Sim & 70 & 87,5 & 25 & 32,1 & 95 & 60,1 & \\
\hline
\end{tabular}

* Teste do Qui-quadrado

Fonte: Autores.

Na Tabela 5 percebeu-se que a grande maioria dos ASBs seguem os protocolos de biossegurança e utilizam frequentemente os EPIs, porém, 58,2\% dos participantes do estudo já apresentou algum acidente com percutâneos. Outra condição refere-se ao não cumprimento dos protocolos após exposição à material biológico por este grupo de profissionais $(56,5 \%)$.

Tabela 5. Distribuição das frequências e análise bivariada entre os fatores relacionados às condutas e práticas profissionais e variável dependente.

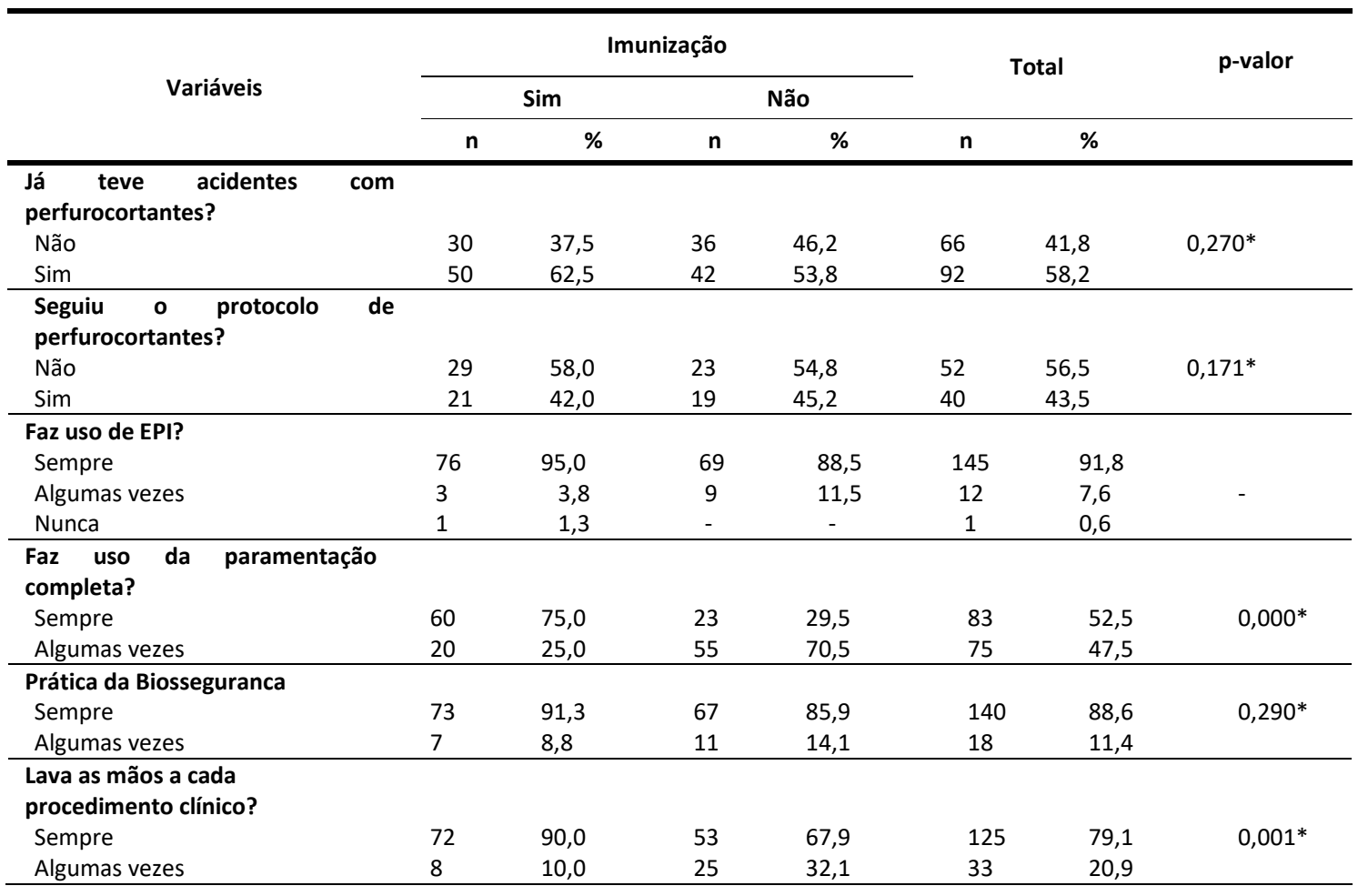

* Teste do Qui-quadrado

Fonte: Autores. 


\section{Discussão}

Com o advento das doenças infectocontagiosas, a contaminação por hepatites virais são agravos em saúde que denotam grande impacto na estrutura epidemiológica (Garbin et al., 2018). Estudos realizados pelo CDC (Centers for Disease Control and Prevention) apontam que a prevalência de casos de infecção pelo VHB é dez vezes mais alta em profissionais da saúde do que na população geral (Rossato \& Ferreira, 2012). Dessa forma, a presente investigação é um dos estudos pioneiros realizado no país, com auxiliares em saúde bucal, que utiliza o teste imunocromatográfico para o mapeamento do status da imunização deste público-alvo.

Neste estudo foi possível observar que, apenas $25,94 \%$ dos auxiliares em saúde bucal, imunizados, apresentavam ensino superior e/ou curso técnico na área da saúde. Da mesma forma, em estudos conduzidos na Índia e Brasil, o perfil socioeducacional dos participantes foi semelhante, ressaltando a falta de preparo de grande parcela dos ASBs para o cargo em que lhes foi indicado (Annaporna et al., 2020; Paes et al., 2018). Além disto, evidenciou-se estatisticamente uma associação entre idade e o status de imunização $(\mathrm{p}=0,015)$, podendo ser justificado em razão da idade estar relacionada com o menor tempo de formação, influenciando na postura profissional (Garcia \& Facchini, 2007; Tipple et al.,2013).

Constatou que os profissionais de saúde que não estavam imunizados neste estudo apresentavam desconhecimento de questões elementares referente à Hepatite $\mathrm{B}$, resultando em maiores números de erros, quando comparados com os indivíduos imunes a doença. De acordo com o estudo de Paes et al., isto pode ser explicado em decorrência do crescente número de ASBs sem um embasamento técnico-profissional, associada a uma capacitação falha, pelas escolas formadoras (Paes et al., 2018).

Tendo em vista que a odontologia é uma das áreas críticas e de extrema vulnerabilidade aos patógenos, o conhecimento acerca das infecções virais torna-se imprescindível para uma prática clínica segura e consciente (Carvalho \& Luz, 2018). Na presente investigação, por meio da análise dos dados, tornou-se notório associações estatisticamente significantes entre a falta de imunização e noções acerca da etiologia, vias de transmissão e prevenção da hepatite B. Dentre as variáveis analisadas nesta dimensão, grande parte dos profissionais de saúde que não estavam imunizados, desconheciam o agente etiológico da doença $(87,7 \%)$ e consequentemente, dispunham de informações insuficientes acerca das vias de transmissão $(71,8 \%)$ e prevenção da doença $(70,5 \%)$. Baseado nesta premissa, tais pontos podem ser fundamentados pela ausência de domínio adequado dos ASBs frente aos protocolos de controle de infecção e ao não cumprimento de tais procedimentos durante a prática odontológica (AlDakhil et al., 2019).

Na presente investigação foi possível observar que 45,6\% dos auxiliares em saúde bucal não estavam imunizados contra o VHB. Neste contexto, também foi observada a presença de lacunas no que tange ao status de imunização, evidenciando a falta de discernimento dos profissionais sobre o número de doses e durabilidade da imunização assegurada pela vacina. Tais pontos foram verificados em estudos realizados em diversos países, como, Estados Unidos 44,2\%, Paquistão 42,20\% e Brasil 44,4\%. (Javaid et al., 2020)

A baixa adesão da imunoprevenção por estes profissionais pode ser explicada tendo em vista o desconhecimento da quantidade de doses necessárias para produção de anticorpos. Ademais, grande parcela dos ASBs não se certificou do status de imunização desempenhado pelo teste anti-HBs. Da mesma forma como no estudo de Garcia et al., conduzido com profissionais da saúde, no qual, em sua maioria, não realizaram o teste anti- HBs tendo em vista o confundimento entre o mesmo e o teste para identificar a infecção por Hepatite B (Garcia \& Facchini, 2017; Garbin et al.,2020).

Apesar da vacina ser considerada a principal forma de proteção contra o VHB e no Brasil, sua distribuição na rede pública ser de forma gratuita, o indivíduo estar vacinado não assegura sua imunização contra a hepatite B (Resende et al., 2010). Nos estudos de Leroux-Roels et al., e Zikang et al., a diminuição gradual dos anticorpos deve-se a completude do esquema vacinal, tipo e/ou intervalo de tempo entre as doses da vacina e comportamentos de saúde dos indivíduos (Leroux- 
Roels et al., 1997; Zikang et al., 2019). No que diz respeito aos comportamentos de saúde, estudos ressaltam que os mesmos podem influenciar na produção de anticorpos, como o consumo de bebidas alcoólicas, tabagismo e sedentarismo, reafirmando a relevância da realização do teste anti-HBs, assim como, a necessidade do reforço das doses vacinais (Garbin et al., Mushtaq et al., 2018). Na presente investigação, percebeu-se que a maioria dos ASBs que relataram não terem adquirido imunidade frente à doença, consumiam bebidas alcoólicas, não praticavam atividades físicas e eram tabagistas ratificando os desfechos encontrados nos estudos de Liu et al., e Yang et al, (Liu et al., 2017; Yang et al., 2016).

No que tange as condutas e práticas dos profissionais, verificou-se que grande parcela dos ASBs que não estavam imunizados já sofreu algum acidente com materiais percutâneos. Neste estudo, embora não se tenha encontrado associações estatisticamente significantes entre a variável dependente e os acidentes com estes materiais, tais casualidades foram notadas em outros estudos, que destacaram que independentemente do tempo de trabalho do auxiliar em saúde bucal, estes profissionais já foram expostos à riscos biológicos pelo menos uma vez durante sua atuação profissional no âmbito odontológico (Aldakhill et al., 2019; Souza \& Araújo, 2018).

Outra questão alarmante percebida no estudo, refere-se à falta de adesão aos protocolos que devem ser desempenhados após a exposição à materiais perfurocortantes. Assim como sugere um estudo, onde tal fato pode ser explicado considerando que grande parte dos auxiliares em saúde ainda infringem as recomendações de entidades de saúde quanto às condutas profissionais, em decorrência da ausência de mecanismos efetivos de controle e fiscalização das ações em saúde suscitando na omissão e imprudência no âmbito odontológico (Garbin et al., 2020).

Considerando a vulnerabilidade dos profissionais da saúde e a necessidade de minimizar os infortúnios ocupacionais, o CDC instaurou as chamadas Precauções Universais. Tais recomendações consistem na utilização adequada dos EPI's (Equipamentos de Proteção Individual), lavagem das mãos antes e após procedimentos clínicos, além da imunização, especialmente para a prevenção das doenças infectocontagiosas (Martins et al., 2017). Neste estudo, ainda que grande parte dos profissionais referenciaram a aplicabilidade das normas e diretrizes de biossegurança e uso rotineiro dos EPI's, percebeu-se que o hábito de lavagem de mãos antes e após os procedimentos, bem como, uso da paramentação por completo, não se fizeram de forma frequente por parte de alguns auxiliares em saúde bucal. Neste contexto, mesmo após o reconhecimento do axioma de biossegurança na prática profissional, e do cenário atual de pandemia, faz-se necessário conscientizar os profissionais da saúde para a importância e a necessidade da adoção de medidas prévias de controles de infecção (Martins et al., 2017; Bizzoca et al.,2020).

Com base neste cenário, ressalta-se que os desafios no que concerne a Hepatite B vão além da investigação e controle de índices epidemiológicos, estando imbuídos nas potencialidades e incentivos públicos para o enfrentamento da doença, prevenção e o autocuidado em saúde, fortalecendo ensino através de discussões acerca das doenças infectocontagiosas de grande impacto para o cenário odontológico.

\section{Conclusão}

Conclui-se que uma expressiva parcela de auxiliares em saúde bucal, atuantes na Atenção Primária à Saúde não estavam imunes, encontrando-se vulneráveis à contaminação pela hepatite B. Ademais, ficou evidente algumas lacunas no que se refere ao conhecimento dos profissionais sobre a doença, sendo que os comportamentos individuais, a ausência da completude do esquema vacinal e teste anti-HBs e as condutas práticas foram fatores que apresentaram influência sobre o status de imunização. 


\section{Agradecimentos}

O presente trabalho foi realizado com apoio da Coordenação de Aperfeiçoamento de Pessoal de Nível Superior- Brasil (CAPES) - Código de Financiamento 001

\section{Referências}

Alavian, S. M., Mahboobi, N., \& Mahboobi, N. (2011) Anti-HBs antibody status and some of its associated factors in dental health care workers in Tehran University of Medical Sciences: Anti-HBs Ab and associated factors in dental society. Hepatitis Monthly, 11(2):99-102.

AlDakhil, L., Yenugadhati, N., Al-Seraihi, O., \& Al-Zoughool, M. (2019). Prevalence and associated factors for needlestick and sharp injuries (NSIs) among dental assistants in Jeddah, Saudi Arabia. Environmental Health and Preventive Medicine, 24(1), 60.

Annapoorna, H. B., Datta, D., Paul, G.T., Rai, N., Sharma, S., \& Kohli, S. (2020). Assessment of the qualification and performance of the assistants working in the private dental clinics in Bilaspur, Chhattisgarh. International Journal of Preventive and Clinical Dental Research, 7(3), 49.

Bizzoca, M. E., Campisi, G., \& Lo Muzio, L. (2020). Covid-19 pandemic: What changes for dentists and oral medicine experts? A narrative review and novel approaches to infection containment. International Journal of Environmental Research and Public Health, 17(11), 3793.

Brasil. Ministério da Saúde. (2006). Portaria n ${ }^{\circ} 399$, de 22 de fevereiro de 2006. Divulga o pacto pela saúde 2006- consolidação do SUS e aprova as diretrizes operacionais do referido pacto. Diário Oficial da União.

Carvalho, T. S., \& Luz, R. A. (2018). Acidentes biológicos com profissionais da área da saúde no Brasil: uma revisão da literatura/Biological accidents with health care workers in Brazil: a literature review. Arquivos Médicos dos Hospitais e da Faculdade de Ciências Médicas da Santa Casa de São Paulo, 63(1), $31-36$.

Garbin, C. A. S., Vanzo, K. L. T., Moimaz, S. A. S., Garbin, A. J. I., \& Chiba, F. Y. (2020). Dental auxiliary hepatitis B immunization: a cross-sectional study in the São Paulo state public health system, Brazil, 2018. Epidemiologia e Serviços de Saúde, 29, e2019113.

Garbin, C. A. S., Wakayama, B., Saliba, T.A, Saliba Junior, O.A., \& Garbin, A.J.I. (2020). A cross-sectional study on dental surgeons' immune status against hepatitis B virus in the Public Health System. Revista do Instituto de Medicina Tropical de São Paulo, 62 , e18.

Garbin, C. A. S., Wakayama, B., Saliba, T.A., Saliba, O., \& Garbin, A.J.I. (2018). Discriminación y prejuicio. La influencia del VIH/SIDA y la Hepatitis B en la actitud de los académicos en odontología. Revista Ciencias de la Salud, 16(2), 279-293.

Garcia, L. P., \& Facchini, L. A. (2008). Vacinação contra a hepatite B entre trabalhadores da atenção básica à saúde. Cadernos de Saúde Pública, 24, 11301140 .

Gomes, A. M., Sousa, T. F., Silva, L. L. D., Carvalho, Y. C. G., \& Filgueira, M. J. P. (2020) Hepatites virais: uma análise clínico-epidemiológica no Estado do Tocantins nos últimos 18 anos. Revista de Patologia do Tocantins, 7(2), 107-113.

Javaid, M., Jamil, M., \& Sajid, M. (2020). Status of vaccination against hepatitis B among dental assistants of multan. Journal of The Pakistan Dental Association, 29(1):42-45.

Jouneghani, A. S., Chaleshtori, M. H., Khosdhdel, A., Kheiri, S., Farrokhni, E., \& Khalafian, P. (2017). Evaluation of response to hepatitis B vaccine in Iranian 6-18-year-old students. Journal of Research in Medical Sciences, 22, 116.

Leroux-Roels, G., Desombere, I., Cobbaut, L., Petit, M.A., Desmons, P., \& Hauser, P. (1997). Hepatitis B vaccine containing surface antingen and selected preS1 and preS2 sequences- Immunogenicity in poor responders to hepatites B vaccines. Vaccine, 15(16), 1732-6.

Liu, F., Guo, Z., \& Dong, C. (2017). Influences of obesity on the immunogenicity of Hepatitis B vaccine. Human Vaccines \& Immunotherapeutics, 13(5), 1014-1017.

Marconi, M. A., \& Lakatos, E.M. (2003). Fundamentos de metodologia científica. 5. ed.-São Paulo: Editora Atlas

Martins, R. J, de Melo, N. B., Garbin, C. A. S., Garbin, A. J. I., \& Domingues, M. K. (2017). O Reencape de Agulhas e Descarte de Resíduos Odontológicos do Grupo E por Estudantes de uma Universidade Pública Brasileira. Ciencia \& Trabajo, 19(59), 91-94.

Mushtaq, N., Awan, M. H., Ali, A., Khalid, S., \& Awan, M. H. (2018). Prevalence of hepatitis B vaccination status among dentists and dental assistants. Pakistan Oral \& Dental Journal, 38(3), 327-329.

Paes, G. R., Ramos, J. T., Ronsani, M. M., \& Meira, T. M. (2018). Formação profissional e conhecimento sobre biossegurança de Auxiliares de Saúde dos setores públicos e privados. Revista da ABENO, 18:43-52

Resende, V. L. S., Abreu, M. H. G., Paiva, S. M., Teixeira, R., \& Pordeus, I. A. (2010). Concerns regarding hepatitis B vaccination and post-vaccination test among Brazilian dentists. Virology Journal, 7, 154.

Rossato, E. M., \& Ferreira, J. (2012). Acidentes com perfurocortantes e cobertura vacinal contra hepatite B entre trabalhadores da Saúde no Município de Santa Rosa, Estado do Rio Grande do Sul, Brasil, 2008. Epidemiologia e Serviços de saúde, 21(3), 487-496.

São Paulo (Estado). (2006). Decreto n ${ }^{\circ}$ 51.433, de 28 de dezembro de 2006. Cria unidade na Coordenadoria de Regiões de Saúde, da Secretária de Saúde, altera a denominação e dispõe sobre a reorganização das Direções Regionais de Saúde. Diário Oficial do Estado de São Paulo. 
Research, Society and Development, v. 10, n. 4, e44810918242, 2021

(CC BY 4.0) | ISSN 2525-3409 | DOI: http://dx.doi.org/10.33448/rsd-v10i9.18242

Souza, F. D. O., \& Araújo, T. M. D. (2018). Exposição ocupacional e vacinação para hepatite B entre trabalhadores da atenção primária e média complexidade. Revista Brasileira de Medicina do Trabalho, 16(1), 36-43.

Souza, F. O., Freitas, P. S. P., Araújo, T. M, \& Gomes, M. R. (2015). Hepatitis B and Anti-HBS vaccination among health workers. Cadernos Saúde Coletiva, 23(2), 172-179.

Teixeira, B. S., Matias, G. L., Xavier, B. L. Q., Moreira, M. R. C., \& Assis, L. M. (2020) Perfil vacinal e sorológico para hepatite B de acadêmicos de enfermagem. Brazilian Journal of Development, 6(11), 86310- 86327.

Tipple, A. F. V., Silva, E. A. C., Teles, S. A., Mendonça, K. M., Souza, A. C. S., \& Melo, D. S. (2013). Acidente com material biológico no atendimento préhospitalar móvel: realidade para trabalhadores da saúde e não saúde. Revista Brasileira de Enfermagem, 66, 378-384.

Wakayama, B., Garbin, C. A. S., Garbin, A. J. I., Saliba, T. A., \& Garbin, A. J. I. (2019). Hepatitis B immunization analysis: tracking of antibody levels among dental patients. The Journal of Contemporary Dental Practice, 20(1), 13-19.

World Health Organization. (2015). Guidelines for the prevention, care and treatment of pearsons with chronic hepatitis B infection.

World Health Organization. (2017). Guidelines on hepatitis B and C testing.

Yang, S., Tian, G., Cui, Y., Ding, C., Deng, M., \& Yu, C. (2016) Factors influencing immunologic response to hepatitis B vaccine in adults. Scientific Reports, 6,27251

Yuan, Q., Wang, F., Zheng, H., Zhang, G., Miao, N., Sun, X., \& Cui, F. (2019). Hepatitis B vaccination coverage among health care workers in China. PloS one, 14(5), e0216598.

Zikang, W.u., Hongdan, Bao., Jun, Yao., Yongdi, Chen., Shunshun, Lu., \& Jing, Li. (2019). Suitable hepatitis B vaccine for adult immunization in China: a systematic review and meta-analysis. Hum Vaccin Immunother, 15 (1), 220-227. 